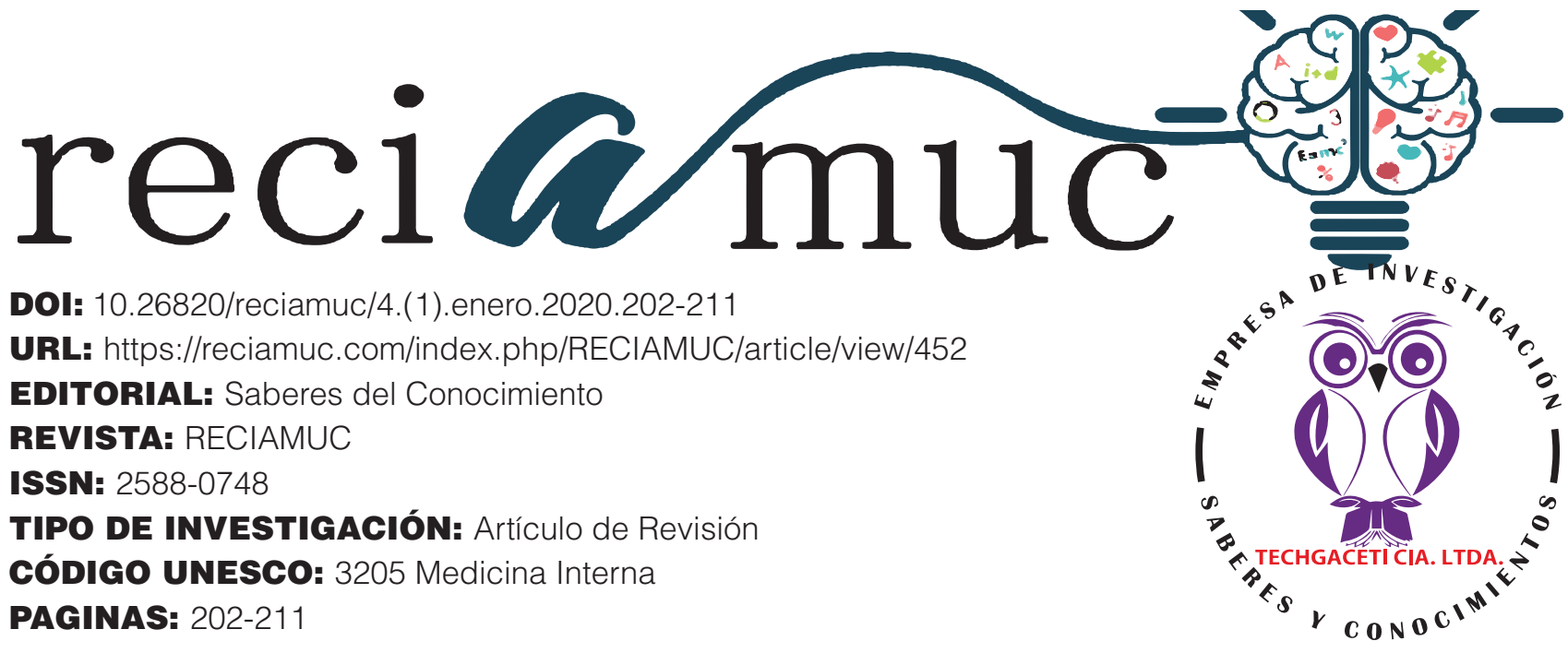

\title{
Cambios electrocardiográficos en hyperkalemia
}

Electrocardiographic changes in hyperkalemia Alterações eletrocardiográficas na hipercalemia

\begin{abstract}
Andrea Isabel Cisneros Jurado; Nadia Alejandra Ugarte Palacios²; José Ignacio Macias Alcivar³; Elen Fausto Suárez Véliz ${ }^{4}$
\end{abstract}

RECIBIDO: 18/11/2019 ACEPTADO: 20/12/2019 PUBLICADO: 31/01/2020

1. Médico; Investigador Independiente; Guayaquil, Ecuador; andreacisneros6@gmail.com; (D) https://orcid.org/0000-0001-6302-602X

2. Médico; Investigador Independiente; Guayaquil, Ecuador; nadia.ugarte@gmail.com; (D) https://orcid.org/00000002-8557-2272

3. Médico; Investigador Independiente; Guayaquil, Ecuador; joseig.89escritor@hotmail.com; (D) https://orcid. org/0000-0003-1093-2779

4. Médico; Investigador Independiente; Guayaquil, Ecuador; esuarezvzs5@gmail.com; (D) https://orcid.org/00000003-1575-8685

CORRESPONDENCIA

Andrea Isabel Cisneros Jurado

andreacisneros6@gmail.com

Guayaquil, Ecuador

๑) RECIAMUC; Editorial Saberes del Conocimiento, 2020 


\section{RESUMEN}

La hiperpotasemia es uno de los trastornos electrolíticos más graves que se observan en la medicina de urgencia. Se identifica cuando el nivel plasmático de potasio es mayor de 5,5 mEq/L. Los niveles elevados de potasio sérico no manifiestan síntomas pero se asocian con cambios en el electrocardiograma en la mayoría de los casos, si no se detecta y trata puede conducir a arritmias cardíacas mortales. La hiperpotasemia puede deberse a tres factores principales: aumento en la ingesta (oral o parenteral), disminución de la excreción renal de potasio y desplazamiento del potasio intracelular al líquido extracelular (redistribución). Las manifestaciones clínicas son principalmente por trastornos de la conducción cardíaca y de la función neuromuscular. El electrocardiograma es la mejor herramienta para valorar la cardiotoxicidad de la hiperpotasemia. Los trastornos electrocardiográficos asociados a la hiperpotasemia: onda T picuda, índice $T / R>0,75$, bradicardia sinusal, onda P plana. El objetivo del tratamiento consiste en la disminución de la concentración del K+ extracelular evitando el consumo de alimentos y la administración de medicamentos ricos en $\mathrm{K}+$. Para eliminar y controlar el exceso de $\mathrm{K}+$ se pueden emplear: diuréticos, tiazídicos y resina de intercambio de cationes (sulfato de poliestireno sódi$\mathrm{co}$ ), este último favorece al intercambio de $\mathrm{K}+$ por el $\mathrm{Na}+$ a nivel gástrico, se suele administrar por vía oral mezclado con sorbitol para evitar el estreñimiento. La metodología usada es descriptiva, con un enfoque documental, es decir, revisar fuentes disponibles en la red, como google académico, con contenido oportuno, actualizado y relevante desde el punto de vista científico que enriquezca el análisis del tema planteado en este artículo.

Palabras clave: Hiperpotasemia, K+, Diurético, Electrocardiograma, Arritmia, Bradicardia sinusal, Onda P plana.

\section{ABSTRACT}

Hyperkalemia is one of the most serious electrolyte disorders seen in emergency medicine. It is identified when the plasma potassium level is greater than $5.5 \mathrm{mEq} / \mathrm{L}$. Elevated levels of serum potassium do not show symptoms but are associated with changes in the electrocardiogram in most cases, if it is not detected and treated it can lead to fatal cardiac arrhythmias. Hyperkalemia can be due to three main factors: increased intake (oral or parenteral), decreased renal excretion of potassium and displacement of intracellular potassium to extracellular fluid (redistribution). The clinical manifestations are mainly due to disorders of cardiac conduction and neuromuscular function. The electrocardiogram is the best tool to assess the cardiotoxicity of hyperkalemia. Electrocardiographic disorders associated with hyperkalemia: peak T wave, T/ $\mathrm{R}$ index $>0.75$, sinus bradycardia, flat $\mathrm{P}$ wave. The goal of treatment is to reduce the concentration of extracellular $\mathrm{K}+$ by avoiding food consumption and the administration of medicines rich in $\mathrm{K}+$. To eliminate and control the excess of $\mathrm{K}+\mathrm{can}$ be used: diuretics, thiazides and cation exchange resin (sodium polystyrene sulfate), the latter favors the exchange of $\mathrm{K}+$ for $\mathrm{Na}+$ at gastric level, it is usually administered orally mixed with Sorbitol to prevent constipation. The methodology used is descriptive, with a documentary approach, that is, reviewing sources available on the web, such as google scholar, with timely, updated and scientifically relevant content that enriches the analysis of the topic raised in this article.

Keywords: Hyperkalemia, K +, Diuretic, Electrocardiogram, Arrhythmia, Sinus bradycardia, Flat P wave.

\section{RESUMO}

A hipercalemia é um dos distúrbios eletrolíticos mais graves observados na medicina de emergência. É identificado quando o nível plasmático de potássio é superior a $5,5 \mathrm{mEq} / \mathrm{L}$. Níveis elevados de potássio sérico não apresentam sintomas, mas estão associados a alterações no eletrocardiograma na maioria dos casos, se não for detectado e tratado, pode levar a complicações cardíacas fatais. arritmias. A hipercalemia pode ser causada por três fatores principais: aumento da ingestão (oral ou parenteral), diminuição da excreção renal de potássio e deslocamento do potássio intracelular para o líquido extracelular (redistribuição). As manifestações clínicas são principalmente devidas a distúrbios da condução cardíaca e função neuromuscular. O eletrocardiograma é a melhor ferramenta para avaliar a cardiotoxicidade da hipercalemia. Distúrbios eletrocardiográficos associados à hipercalemia: onda T pico, índice T / R> 0,75, bradicardia sinusal, onda P plana. O objetivo do tratamento é reduzir a concentração de $\mathrm{K}+$ extracelular, evitando o consumo de alimentos e a administração de medicamentos ricos em $\mathrm{K}+$. Para eliminar e controlar o excesso de $\mathrm{K}+$ podem ser utilizados: diuréticos, tiazidas e resina de troca catiônica (sulfato de poliestireno de sódio), este último favorece a troca de $\mathrm{K}+$ por $\mathrm{Na}+\mathrm{no}$ nível gástrico, geralmente é administrado por via oral misturado com sorbitol para prevenir a constipação. A metodologia utilizada é descritiva, com abordagem documental, ou seja, revisando fontes disponíveis na web, como o google scholar, com conteúdo oportuno, atualizado e cientificamente relevante que enriquece a análise do tópico levantado neste artigo.

Palavras-chave: Hipercalemia, K +, Diurético, Eletrocardiograma, Arritmia, Bradicardia sinusal, Onda P plana. 


\section{Introducción}

La hiperpotasemia se define como la presencia de una concentración plasmática de potasio superior a 5,5 mEq/l. Constituye la alteración electrolítica más importante por su potencial gravedad pudiendo ocasionar alteraciones en la conducción cardiaca y arritmias potencialmente letales. Es un problema prevalente en la práctica clínica y forma parte de los trastornos del medio interno que se asiste con más frecuencia en los servicios de urgencia.

La disminución de la excreción renal de potasio es la causa más frecuente de esta alteración, habitualmente secundaria a injuria renal aguda, enfermedad renal crónica o bloqueo farmacológico del eje renina-angiotensina-aldosterona. Puede responder a una alteración en la distribución corporal del potasio, por movimiento del ion al espacio extracelular, situación que puede verse aún con un potasio corporal total descendido. Una causa menos frecuente es el aporte aumentado de potasio en la dieta o de forma parenteral.

Las manifestaciones clínicas que revisten mayor gravedad son las alteraciones en la conducción cardíaca y la parálisis muscular. Habitualmente cuando existen síntomas musculares se pueden evidencian cambios electrocardiográficos característicos. Estas manifestaciones severas se vinculan a niveles séricos de potasio $\geq 7 \mathrm{mEq} / \mathrm{l}$ en pacientes con hiperpotasemia crónica, o a niveles inferiores en hiperpotasemias de instalación aguda (Boada-Pippo, Dic. iembre 2012). La afectación muscular es en general ascendente comprometiendo inicialmente miembros inferiores con progresión a tronco y miembros superiores. La afectación de pares craneanos y músculos respiratorios es inhabitual. En casos graves la debilidad evoluciona a parálisis flácida que puede confundirse con un Guillan Barre. En este artículo se explicara con más detalle el diagnostico, etiología y tratamiento de este desequilibrio.

\section{Metodología}

Esta investigación está dirigida al estudio "Cambios electrocardiográficos en hyperkalemia". Para realizarlo se usó una metodología descriptiva, con un enfoque documental, es decir, revisar fuentes disponibles en la red, como google académico, con contenido oportuno y relevante desde el punto de vista científico para dar respuesta a lo tratado en el presente artículo y que sirvan de inspiración para realizar otros proyectos. Las fuentes consultadas pueden ser estudiadas al final, en la bibliografía.

\section{Resultados}

La hiperpotasemia es uno de los trastornos electrolíticos más graves que se observan en la medicina de urgencia. Se identifica cuando el nivel plasmático de potasio es mayor de $5,5 \mathrm{mEq} / \mathrm{L}$. Se detecta en 1-10\% de los pacientes hospitalizados según estudios realizados en Estados Unidos, Japón y China. Los niveles elevados de potasio sérico no manifiestan síntomas pero se asocian con cambios en el electrocardiograma en el $46 \%$ de los casos (Peralta-Codas, Marzo 2019). Si no se detecta y trata puede conducir a arritmias cardíacas mortales.

La hiperpotasemia puede deberse a tres factores principales:

- Aumento en la ingesta (oral o parenteral).

- Disminución de la excreción renal de potasio.

- Desplazamiento del potasio intracelular al líquido extracelular (redistribución). 
Tabla 1. Clasificación de la hiperpotasemia

\begin{tabular}{|l|l|}
\hline \multicolumn{1}{|c|}{ Nivel } & \multicolumn{1}{c|}{ Medición } \\
\hline Leve & $5,0<[\mathrm{K}+]<5,9 \mathrm{mEq} / \mathrm{L}$ \\
\hline Moderada & $6,0<[\mathrm{K}+]<6,4 \mathrm{mEq} / \mathrm{L}$ \\
\hline Severa & {$[\mathrm{K}+] \geq 6,5 \mathrm{mEq} / \mathrm{L}$} \\
\hline Fatal & {$[\mathrm{K}+] \geq 10,0 \mathrm{mEq} / \mathrm{L}$} \\
\hline
\end{tabular}

Fuente: (Ovalles-Mena, 2017)

En condiciones normales el 98\% del potasio en el cuerpo se encuentra dentro de las células de varios tejidos, mientras que solo alrededor del $2 \%$ está circulando en la sangre. La hiperpotasemia puede ser causada por una excreción renal minimizada, una ingestión excesiva o una fuga de potasio del espacio intracelular (Peralta-Codas, Marzo
2019). La disminución de la excreción renal de potasio es la causa más frecuente de esta alteración, habitualmente secundaria a injuria renal aguda, enfermedad renal crónica o bloqueo farmacológico del eje renina-angiotensina-aldosterona. La siguiente tabla resume las principales causas de hiperpotasemia:

Tabla 2. Causas de la Hiperpotasemia

\footnotetext{
Pseudohiperpotasemia

Muestra hemolizado.

Leucocitosis (>200 000/ $\mu \mathrm{L})$.

Trombocitosis intensa $(>500000 / \mu \mathrm{L})$.

Torniquete excesivamente apretado o contracción muscular de la extremidad.

Aporte excesivo de potasio oral o intravenoso

Soluciones parenterales.

Ingesta oral en jugos y suplementos orales de potasio.

Disminución de la eliminación renal Insuficiencia renal aguda o crónica

Enfermedad de Addison.

Hiperplasia suprarrenal congénita.

Hipoaldosteronismo, hiporreninémico, acidosis tubular renal tipo IV.

Fármacos que interfieren con la liberación y/o secreción de aldosterona: IECA, ARA II, inhibidores de la renina, heparina, ciclosporina, tacrolimus, sobredosis de digital. Fármacos que inhiben la secreción renal de potasio: diuréticos ahorradores de potasio. (espironolactona, eplerenona, triamtereno, amilorida), trimetoprima, pentamidina, L-arginina.

Disfunción tubular distal (mieloma, amiloidosis, lupus, trasplante renal, drepanocitosis).

Paso de potasio al líquido extracelular

Acidosis

Lisis celular: traumatismos extensos, quemaduras, lisis tumoral, rabdomiólisis y hemólisis.

Parálisis periódica hiperpotasemia.
}

Fuente: (Boada-Pippo, 2012) 
El $75 \%$ de los casos de hiperpotasemia en pacientes internados son inducidos por drogas ahorradoras de potasio. La hiperpotasemia $\geq 6 \mathrm{mmol} / \mathrm{L}$ se observa en el $1 \%$ de los consumidores de inhibidores de la enzima convertidora de la angiotensina I y en $2,8 \%$ de los usuarios de antagonistas de los receptores de la angiotensina II. La combinación de medicamentos ahorradores de potasio multiplica el riesgo de este evento farmacológico adverso. Las tasas de mortalidad en pacientes con hiperpotasemia superior a $7,0 \mathrm{mmol} / \mathrm{L}$ alcanzan el $67 \%$ si no se corrige oportunamente (Peralta-Codas, Marzo 2019).

En pacientes portadores de marcapasos cardíacos, la hiperpotasemia puede causar diversos tipos de disfunción del dispositivo, con consecuencias muy graves. En esta situación, el exceso de potasio extracelular aumenta el potencial de reposo de la membrana celular miocárdica. Si este potencial supera la energía emitida por el marcapasos según su programación, la despolarización del miocardiocito queda inhibida. Esta situación se reconoce en el electrocardiograma por la ausencia de la despolarización tras la espícula. Su diagnóstico clínico es la asistolia (Pere-Valles, Febrero 2013).

La presencia de un marcapasos cardíaco no siempre protege al miocardio de los efectos deletéreos de la hiperpotasemia. Es importante saber que el dispositivo precisa de un equilibrio electrolítico normal para su buen funcionamiento. La monitorización del ritmo cardíaco, obligada en toda hiperpotasemia sintomática, no debe obviarse en pacientes portadores de marcapasos. La presencia de un marcapasos no debe condicionar la indicación de hemodiálisis urgente en una hiperpotasemia grave (Fernández-Alvarez, 2015).

\section{Etiología}

1. Retención renal de potasio, se manifiesta en:

a) La insuficiencia renal aguda o crónica, donde el filtrado glomerular desciende por debajo de los 5-10 ml/min., ocasionando retención de $\mathrm{K}+$ por vía renal, estimulando la secreción de aldosterona, aumentando la secreción de potasio por las nefronas funcionales, condicionando de tal forma una hiperpotasemia.

b) Hipoaldosteronismo: la aldosterona es una hormona de origen suprarrenal cuyas funciones principales son las de defender al organismo contra la hipovolemia y prevenir la hiperpotasemia, al verse disminuida en su secreción, se reduce la excreción de potasio vía renal, produciendo hiperpotasemia a nivel extracelular. El hipoaldosteronismo familiar primario, de origen congénito, que se presenta por defecto de las hormonas corticosterona-metiloxidasa I o II, manifestando antes de los 3 primeros meses de vida, puede pasar desapercibido hasta producirse una disminución de volumen potásico.

c) Fármacos: van relacionados a la hiperpotasemia por la obstrucción en el conjunto renina-aldosterona, como ocurre con la heparina que además de su acción anticoagulante inhibe la síntesis de la aldosterona y predispone al desarrollo de hiperpotasemia. La administración de diuréticos como la Amilorida, reduce la reabsorción distal del sodio y la secreción de hidrogeniones y $\mathrm{K}+$, ocasionando acidosis metabólica hiperclorémica e hiperpotasémica.

2. Redistribución transcelular, se refiere a que se producen alteraciones del $\mathrm{pH}$ extracelular, con acidosis metabólica, lo que conduce el potasio desde el espacio intracelular al extracelular por el descenso del $\mathrm{pH}$, induciendo al aumento de la concentración plasmática del $\mathrm{K}+$ mayor a $5.5 \mathrm{mEq} / \mathrm{l} .4$ Este proceso se presenta en:

a) La acidosis metabólica, donde el ingreso de $\mathrm{H}+$ a la célula colabora en la salida de $\mathrm{K}+$ intracelular para incrementar los valores de $\mathrm{K}+$ extracelular. La acidosis metabólica inducida por ácidos orgánicos (láctica, cetoacidótica) no produce hiperpotasemia. 
b) La destrucción tisular, es una de las causas frecuentes de hiperpotasemia aguda por salida de K+ intracelular, ocasionada por transfusiones sanguíneas incompatibles, disfunciones valvulares agudas con hemólisis, traumatismos con aplastamiento extenso de tejido, rabdomiólisis, quemaduras y lisis tumoral inducida por quimioterapia por tratamientos del linfoma de Burkitt o de leucemia aguda (Vega-Galabia, Junio 2019).

c) Fármacos: la redistribución transcelular también se puede presentar por inducción de fármacos como los bloqueantes beta, que actúan en el efecto beta-adrenérgico de captación celular de $\mathrm{K}+$, por tanto facilitan el paso del K+ hacia el espacio extracelular.

i. Los Aminoácidos catiónicos son aminoácidos que al ingresar al compartimiento celular provocan una hiperpotasemia severa.

ii. La Succinilcolina, es un miorrelajante que se utiliza en procedimiientos de inducción anestésica, su acción es la de despolarizar al músculo y aumentar su permeabilidad hacia el $\mathrm{K}+$, cambiando la carga eléctrica intracelular, provocando la salida de $\mathrm{K}+$ hacia el medio extracelular, en pacientes con depósitos de $\mathrm{K}+$ aumentados.

iii. En una sobrecarga exógena de $\mathrm{K}+$, si la función renal es normal la hiperpotasemia es rara, sin embargo, si existen alteraciones en la función renal puede producirse hiperpotasemia por ingesta elevada de $\mathrm{K}+$, superior a $160 \mathrm{mEq}$, ocasionando arritmias cardiacas poniendo en peligro la vida del paciente.

iv. Cuando se realiza una excesiva administración de sales de K+, vía intravenosa para la reposición de $\mathrm{K}+$, se debe hacer un control primordial para que este se difunda de manera lenta.

\section{Manifestaciones clínicas}

Son principalmente por trastornos de la conducción cardíaca y de la función neuro- muscular. El electrocardiograma es la mejor herramienta para valorar la cardiotoxicidad de la hiperpotasemia (Pere-Valles, Febrero 2013). Con niveles de alrededor de 6,5 mEq/l aparecen ondas $T$ picudas, y por encima de $7 \mathrm{mEq} / \mathrm{l}$ se prolonga el intervalo PR, se pierde la onda $P$ y más tarde se produce un ensanchamiento del complejo QRS.

Cuando las cifras de potasio superan los 8 $\mathrm{mEq} / \mathrm{l}$, el complejo QRS puede converger con la onda $T$ y formar una onda sinuosa (Sanchez-Higuera, 2016). Pero es fundamental recordar que ésta es una clasificación académica y que con cualquier grado de hiperpotasemia pueden aparecer arritmias ventriculares fatales. En el sistema neuromuscular la hiperpotasemia puede producir parestesias, debilidad muscular e incluso parálisis flácida.

\section{Diagnóstico}

Se debe valorar la concentración extracelular de $\mathrm{K}+$, las alteraciones electrocardiográficas y medir los niveles de aldosterona y renina. La electrocardiografía señala la presencia de ondas $T$ puntiagudas, en concentraciones extracelulares de $\mathrm{K}+$ de 6.5 $\mathrm{mEq} / \mathrm{L}$.; se amplía el intervalo PR, se pierde la onda $P$ y se produce una extensión del complejo QRS en niveles de $\mathrm{K}+$ extracelular de 7 a $8 \mathrm{mEq} / \mathrm{L}$; sin embargo si el $\mathrm{K}+$ extracelular sobrepasa los $8 \mathrm{mEq} / \mathrm{L}$, el complejo QRS se une con la onda T formando una onda sinuosa; por lo cual ocurrirá un paro cardiaco si se presentan valores de $\mathrm{K}+$ mayores a $8 \mathrm{mEq} / \mathrm{L}$ (Camargo, Diciembre 2013).

\section{Alteraciones electrocardiográficas en la hiperpotasemia}

Trastorno electrocardiográfico 


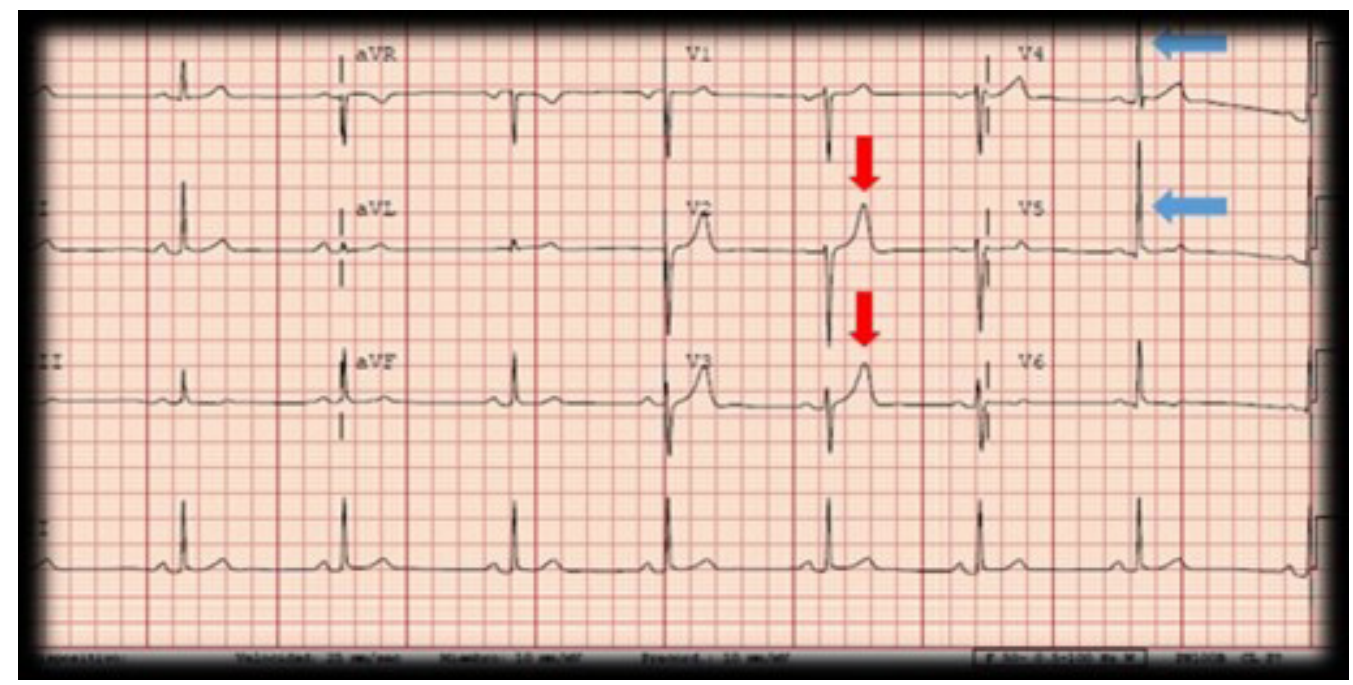

Figura 1. Frecuencia Onda T picuda

Fuente: (Sanchez-Higuera, 2016)

Nota: Hiperpotasemia leve: Se evidencia ondas T picudas, altas, en V3-V6. El intervalo QT está dentro de límites normales.

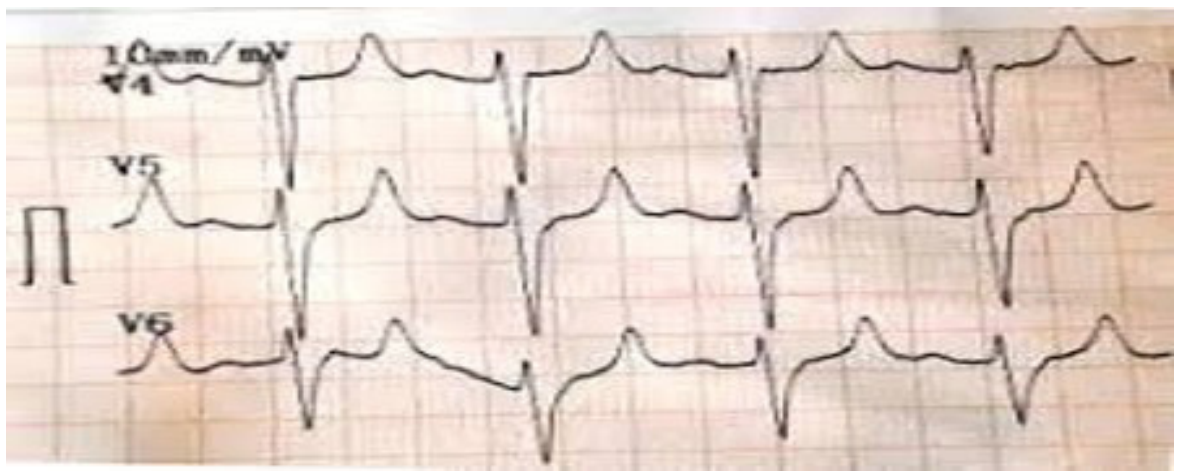

Figura 2. Índice $T / R>0,75$

Fuente: (Peralta-Codas, Marzo 2019)

\section{PAUSA SINUSAL}

- Ausencia de onda P

- Lo que determina una interrupción del ritmo sinusal.

- El nódulo seno-auricular no inicia los impulsos eléctricos, en cambio otra parte del sistema de conducción del corazón actúa como marcapasos

ECG NORMAL
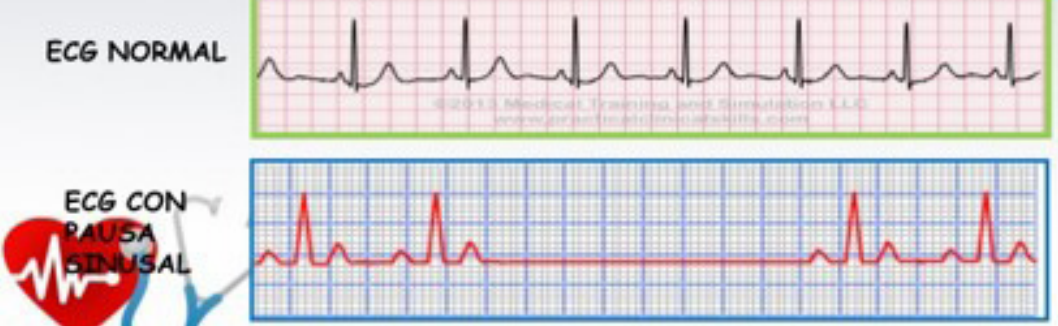

Figura 3. Bradicardia sinusal

Fuente: (Star, 2017) 


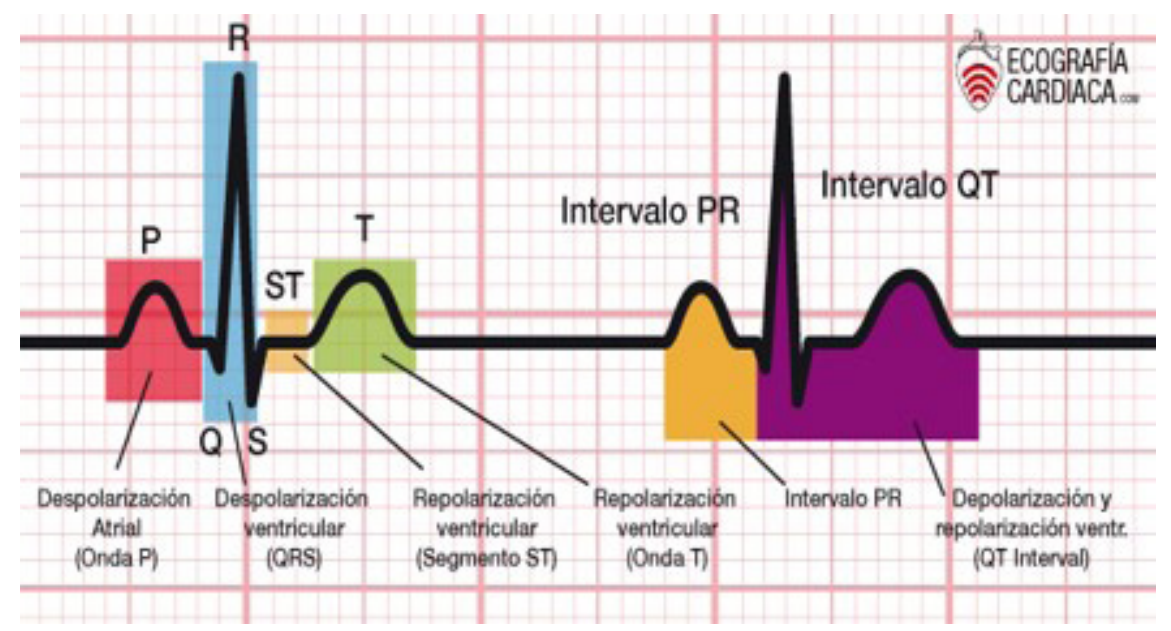

Figura 4. Onda P plana 1

Fuente: (Google Sites, 2017)

Nota: Hiperpotasemia severa: Se observa complejos mucho más anchos y deformados, correspondientes a cifras de $\mathrm{K}+$ de $7,5 \mathrm{mEq} / \mathrm{L}$. Nótese la desaparición de la onda $\mathrm{P}$.

\section{Tratamiento de la hiperpotasemia}

La hiperpotasemia es un trastorno potencialmente mortal, por lo que se debe tratar de forma precoz y eficaz. La presencia de hiperpotasemia junto con alteraciones en el electrocardiograma debe considerarse una emergencia, ya que en cuestión de minutos puede producirse una arritmia fatal. El objetivo del tratamiento consiste en la disminución de la concentración del K+ extracelular evitando el consumo de alimentos y la administración de medicamentos ricos en $\mathrm{K}+$.

Para eliminar y controlar el exceso de $\mathrm{K}+$ se pueden emplear: diuréticos, tiazídicos y resina de intercambio de cationes (sulfato de poliestireno sódico), este último favorece al intercambio de $\mathrm{K}+$ por el $\mathrm{Na}+$ a nivel gástri$\mathrm{co}$, se suele administrar por vía oral mezclado con sorbitol para evitar el estreñimiento. A continuación se mencionan diferentes opciones recomendadas en el manejo de la hiperpotasemia:

- Gluconato de calcio al $10 \%$ : se administra $10 \mathrm{ml}$ vía endovenosa en bolo durante un lapso de 5 minutos, pudiendo repetir los bolos con una duración de la acción entre 30 a 60 minutos. Antagoniza el efecto cardiaco de la hiperpotase- mia.

- Salbutamol: se puede administrar vía endovenosa en dosis de 0,5 mg diluido en $100 \mathrm{ml}$ de solución dextrosa al $5 \%$ administrando durante 15 minutos. Otra opción es la administración vía inhalatoria de 10 a 20 mg (2-4 ml) en nebulización durante 10 minutos. Es un fármaco beta adrenérgico que favorece el desplazamiento de potasio al interior de la célula.

- Insulina + glucosa: se administra 10 unidades de insulina cristalina vía endovenosa junto con solución dextrosa al $10 \%$ (500 ml) o solución dextrosa al 50 \% (50 $\mathrm{ml})$. Con una duración de acción de 6 a 8 horas, la insulina favorece el desplazamiento de potasio al espacio intracelular.

- Bicarbonato sódico: especialmente si existe acidosis se administra bicarbonato $1 / 6 \mathrm{M} 250$ a $500 \mathrm{ml}$ o también $50 \mathrm{ml}$ de bicarbonato $1 \mathrm{M}$. Se administra cada 6 a 8 horas.

- Quelantes intestinales: como el sulfonato de poliestireno sódico 15 a $50 \mathrm{~g}$ (3 a 4 cucharadas disueltas en agua) vía oral cada 4 a 6 horas. Se administra cada 12 horas. Favorece la eliminación de pota-

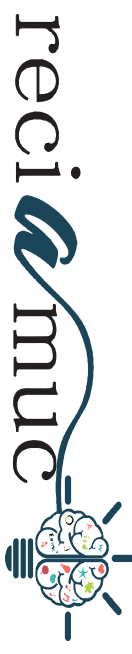


sio a través de las heces.

Diuréticos de asa: como furosemida a dosis de 40 a 200 mg vía iv puede administrarse horario y luego cada 6,8 o 12 horas. Favorece la eliminación renal de potasio. De utilidad siempre que no exista insuficiencia renal severa.

- Diálisis: que puede ser por hemodiálisis o diálisis peritoneal. Es capaz de remover de 25 - $50 \mathrm{~m}$ Eq/hora.

- Resinas de intercambio iónico favorece la eliminación de potasio por el tubo digestivo.

\section{Conclusiones}

La hiperpotasemia se define como la presencia de una concentración plasmática de potasio superior a 5,5 mEq/l. Constituye la alteración electrolítica más importante por su potencial gravedad pudiendo causar alteraciones en la conducción cardiaca y arritmias potencialmente letales. Es un problema prevalente en la práctica clínica y forma parte de los trastornos del medio interno que se asiste con más frecuencia en los servicios de urgencia.

Un electrocardiograma de hiperpotasemia se debe diagnosticar inmediatamente con el fin de instaurar el tratamiento adecuado, debido a que un retraso diagnóstico puede conllevar la muerte del paciente. El cuadro clínico de hiperpotasemia se caracteriza por debilidad que puede evolucionar a parálisis flácida e hipo ventilación, por aumento de la despolarización y disminución de la excitabilidad de la membrana; si bien los efectos más graves son los cardiológicos, con riesgo de fibrilación ventricular y asistolia.

Las alteraciones electrocardiográficas reflejan en realidad el gradiente entre iones de potasio dentro y fuera de las células miocárdicas, y no tanto los niveles de potasio sérico. Inicialmente, con niveles de potasio en torno a 5.5-6.5 mEq/L aparecen ondas $T$ acuminadas, característicamente altas y pi- cudas, con base estrecha, más visibles en las derivaciones II, III y V2-V6.

Si el potasio sigue aumentando hasta 6-6.5 $\mathrm{mEq} / \mathrm{L}$, se produce ensanchamiento de los complejos QRS, que se hacen además irregulares. Con niveles de potasio mayores, de hasta $9 \mathrm{mEq} / \mathrm{L}$ se produce ensanchamiento de las ondas $P$ y aplanamiento de las mismas, con desaparición de la despolarización auricular y aparición de pausas sinusales. También se alarga el intervalo PR y los complejos QRS se hacen aún más anchos, fusionándose con las ondas $T$ y dando un patrón característico de QRS-ST-T en forma de onda sinusoidal, el ST se puede elevar, con niveles mayores de $10 \mathrm{mEq} / \mathrm{L}$ se puede producir fibrilación ventricular.

El objetivo del tratamiento consiste en la disminución de la concentración del K+ extracelular evitando el consumo de alimentos y la administración de medicamentos ricos en $\mathrm{K}+$. Para eliminar y controlar el exceso de $\mathrm{K}+$ se pueden emplear: diuréticos, tiazídicos y resina de intercambio de cationes (sulfato de poliestireno sódico), este último favorece al intercambio de $\mathrm{K}+$ por el $\mathrm{Na}+\mathrm{a}$ nivel gástrico, se suele administrar por vía oral mezclado con sorbitol para evitar el estreñimiento.

\section{Bibliografía}

Boada-Pippo. (2012). Hiperpotasemia severa en emergencia. Archivos de Medicina Interna, 34(3).

Camargo, F. (Diciembre 2013). Hipopotasemia e Hiperpotasemia. Revista de Actualización Clinica Medica Investiga Volumen39, http://www.revistasbolivianas.org.bo.

Fernández-Alvarez. (2015). Hiperpotasemia: Alteraciones electrocardiográficas e identificación dentro de un servicio de urgencias. Obtenido de II Congreso Virtual Internacional SEEUE: http://congresovirtual.enfermeriadeurgencias.com

Google Sites. (2017). Bases electrocardiográficas. Obtenido de Portafolio de cuidados en la $\mathrm{UCI}$ : https://sites.google.com

Ovalles-Mena. (2017). EMERGENCIAS ENDOCRINOLÓGICAS. Caracas - Venezuela: 1era edición.

Peralta, R., Codas Martínez, L., \& Cubilla Zaracho, 
C. (2019). Manifestaciones electrocardiográficas en pacientes con hiperpotasemia del Servicio de Clínica Médica del Hospital Nacional en 2018. Revista Virtual Sociedad de Paraguay Medica, 6(1), 54 - 62. Obtenido de http://scielo. iics.una.py/scielo.php?script=sci_abstract\&pi$\mathrm{d}=$ S2312-38932019000100054\&Ing=en\& $\mathrm{nrm}=\mathrm{i}-$ so\&tlng=es

Pere-Valles. (2013). Disfunción severa de marcapasos por hiperpotasemia. Nefrología (Madr.), 33(3), 443-622. doi:10.3265/Nefrologia.pre2013.

Feb. 11847
Sanchez-Higuera. (2016). Los Casos Clínicos más docentes del año 2015 en Cardiología. Cardio Teca, $23-28$.

Star, S. (17 de Septiembre de 2017). Alteraciones en el electrocardiograma. Obtenido de Slide Share: https://www.slideshare.net

Vega-Galabia. (2019). Epidemiología de la hiperpotasemia en la enfermedad renal crónica. Nefrología Volumen, 39(3), 223-338.

\section{CITAR ESTE ARTICULO:}

Cisneros Jurado, A., Ugarte Palacios, N., Macias Alcivar, J., \& Suárez Véliz, E. (2020). Cambios electrocardiográficos en hyperkalemia. RECIAMUC, 202211. doi:10.26820/reciamuc/4.(1).enero.2020.202-211

\section{(c) $(1) \odot \odot$ BY NC SA}

RECONOCIMIENTO-NOCOMERCIAL-COMPARTIRIGUAL CC BY-NC-SA

ESTA LICENCIA PERMITE A OTROS ENTREMEZCLAR, AJUSTAR Y CONSTRUIR A PARTIR DE SU OBRA CON FINES NO COMERCIALES, SIEMPRE Y CUANDO LE RECONOZCAN LA AUTORÍA Y SUS NUEVAS CREACIONES ESTÉN BAJO UNA LICENCIA CON LOS MISMOS TÉRMINOS. 